\title{
Kainate Receptors Act as Conditional Amplifiers of Spike Transmission at Hippocampal Mossy Fiber Synapses
}

\author{
Shankar Sachidhanandam, ${ }^{1}$ Christophe Blanchet, ${ }^{1}$ Yannick Jeantet, ${ }^{2}$ Yoon H. Cho, ${ }^{2}$ and Christophe Mulle ${ }^{1}$ \\ ${ }^{1}$ Centre National de la Recherche Scientifique (CNRS) Unité Mixte de Recherche (UMR) 5091, Bordeaux Neuroscience Institute, University of Bordeaux, \\ 33077 Bordeaux Cedex, France, and ${ }^{2}$ CNRS UMR 5228, Center for Integrative and Cognitive Neuroscience, University of Bordeaux, 33405 \\ Talence Cedex, France
}

Hippocampal mossy fiber (Mf) synapses are viewed as conditional detonators, assisting CA3 cells in complex network functions. By analyzing mice deficient for GluK2 (GluR6), GluK3 (GluR7) and GluK5 (KA2) genes we show that kainate receptors (KARs) play a crucial role in the control of synaptic integration and spike transmission efficacy at Mf synapses. We dissected out the role of the different KAR functions at Mf synapses and we show that presynaptic and postsynaptic KARs concur to amplify unitary Mf synaptic inputs to trigger spike discharge within a wide range of frequencies (from 1 to $50 \mathrm{~Hz}$ ). Moreover, KARs strongly favor spike transmission in response to patterns of presynaptic activity mimicking in vivo dentate granule cell activity. By amplifying spike transmission, KARs also facilitate the induction of associative long-term potentiation in CA3. Hence the actions of KARs as amplifiers of spike transmission contribute largely to the "conditional detonator" function of Mf synapses and are likely important for spatial information processing.

\section{Introduction}

Synapses between hippocampal mossy fibers (Mf), that arise from dentate granule cells, and CA3 pyramidal cells are a key component of information processing along the hippocampal trisynaptic circuit. Models of hippocampal function have proposed that Mf synapses act as detonator or teacher synapses important for the storage of information and for the retrieval of entire memory patterns from partial or degraded inputs (for review, see Henze et al., 2000; Bischofberger et al., 2006). More recently, it was proposed that Mf synapses are "conditional detonators," dependent on the pattern of activity of presynaptic granule cells (Henze et al., 2002; Bischofberger et al., 2006). It is thus important to understand how granule cell firing patterns influence the activity of CA3 pyramidal cells.

Mf-CA3 synapses are endowed with a number of unique features, including prominent short-term plasticity (Henze et al., 2000; Nicoll and Schmitz, 2005) that is crucial in processing complex presynaptic activity. Ionotropic glutamate receptors of the kainate type (KARs) potentially influence information transfer at Mf-CA3 synapses in at least three different ways. Presynaptically, KARs activated by a single release event facilitate subsequent transmitter release likely through the activation of GluK2/GluK3 heteromers (Contractor et al., 2003; Lerma, 2006; Pinheiro et al., 2007) (GluK1, GluK2, GluK3,

Received Dec. 5, 2008; revised Feb. 16, 2009; accepted March 9, 2009

This work was supported by grants from the Centre National de la Recherche Scientifique, the Ministère de la Recherche of France, the Conseil Régional d'Aquitaine, the European Commission (contract LSH-2004-019055), the Agence Nationale de la Recherche (ANR contract PreSynGlu) to C.M., and the Fondation pour la Recherche Medicale to S.S. We would like to thank György Buzsáki for kindly providing us with the physiological granule cell firing patterns, and David Perrais, Paulo Pinheiro, and Nelson Rebola for critical reading of this manuscript.

Correspondence should be addressed to Christophe Mulle at the above address. E-mail: mulle@u-bordeaux2.fr. D01:10.1523/JNEUROSCI.5807-08.2009

Copyright $\odot 2009$ Society for Neuroscience $\quad 0270-6474 / 09 / 295000-09 \$ 15.00 / 0$ and GluK5, new International Union of Basic and Clinical Pharmacology nomenclature for GluR5, GluR6, GluR7, and KA2, respectively). This leads to an increased extent of shortterm plasticity in response to various patterns of repetitive stimulation. Postsynaptically, mossy fiber EPSCs (Mf-EPSCs) display a KAR component of small amplitude and slow decay kinetics (Castillo et al., 1997) that favors temporal summation of synaptic signals, as proposed for CA1 interneurons (Frerking and Ohliger-Frerking, 2002). Finally, through a metabotropic action that requires the GluK5 subunit, synaptic activation of postsynaptic KARs reversibly inhibits a $\mathrm{Ca}^{2+}$-activated $\mathrm{K}^{+}$current responsible for the slow afterhyperpolarizing potential ( $\left.I_{\text {sAHP }}\right)$ (Melyan et al., 2002; Ruiz et al., 2005). Thus, a short train of stimulation to the mossy fibers not only directly depolarizes the postsynaptic membrane but, in addition, increases neuronal excitability for several seconds.

Despite these well described actions of KARs at the Mf-CA3 synapse, how they may participate to the conditional detonator function of Mf synapses has not been investigated. To address this question, we have studied the influence of KARs in shaping the Mf-EPSP waveform and in controlling the short-term dynamics of synaptic integration. In the absence of suitable pharmacological tools, we took advantage of mice deficient for the KAR subunits GluK2, GluK3 and GluK5, which are differentially affected in the functional properties of Mf synapses: (1) the presynaptic action of KARs is lost both in GluK2 ${ }^{-1-}$ and GluK3 ${ }^{-1-}$ mice (Contractor et al., 2001; Pinheiro et al., 2007); (2) the KAR component of Mf-EPSCs is absent in GluK2 $2^{-1-}$ mice, but preserved in GluK3 ${ }^{-1-}$ mice (Mulle et al., 1998) and displays faster decay kinetics in GluK5 ${ }^{-1-}$ mice (Contractor et al., 2003) and (3) the metabotropic action of KARs is absent in GluK5 ${ }^{-1-}$ mice (Ruiz et al., 2005). Our results indicate that KARs strongly control the efficacy of spike transmission between presynaptic granule cells 

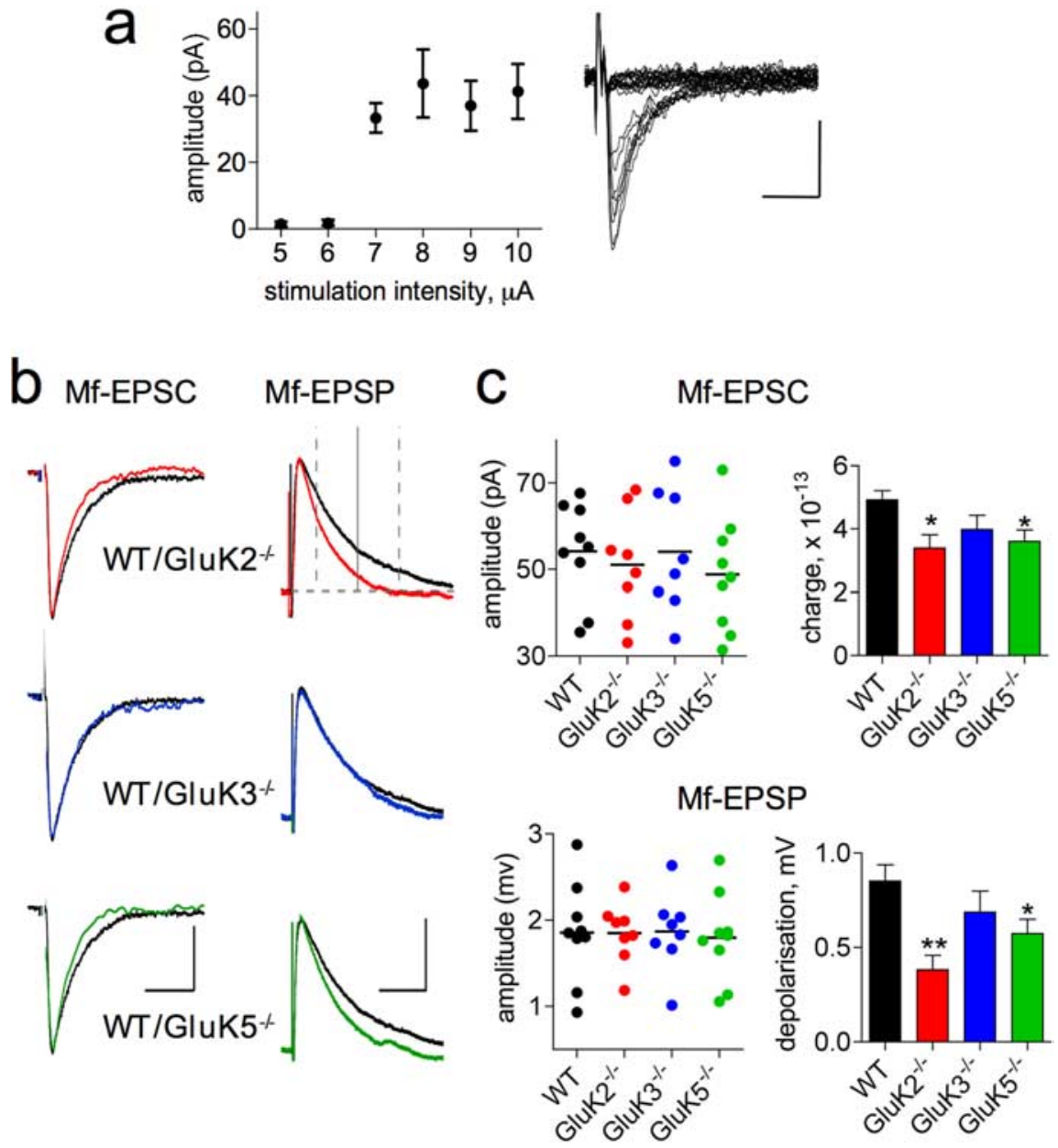

Figure 1. KARs shape unitary Mf-EPSCS and Mf-EPSPs. $\boldsymbol{a}$, Mf-EPSC amplitude (left) as a function of stimulation intensity reveals the minimal stimulation needed to record from a single Mf synapse. Superimposed recordings (right) during increasing intensity of stimulation showing "all or none" unitary Mf-EPSCs. $\boldsymbol{b}$, Superimposed averaged unitary Mf-EPSCs (left) and corresponding averaged Mf-EPSPs (right) of 15 traces obtained from WT (black) and GluK2 ${ }^{-1-}$ (red), GluK3 ${ }^{-1-}$ (blue), and GluK5 $^{-1-}$ (green) mice. This color code is kept in all figures. In the top left panel, the timeframe of $28-121$ ms after stimulation (vertical dotted gray lines) highlights the range where an ANOVA analysis showed a significant difference in Mf-EPSP waveform between WT and GluK2 ${ }^{-1-}$ mice (see Results). Residual Mf-EPSP depolarization at $65 \mathrm{~ms}$ after the stimulus (vertical whole line) was used as an indication of EPSP decay kinetics (the horizontal dotted gray line corresponds to the resting membrane potential level). Calibrations: $10 \mathrm{~ms}$ and 20 pA for Mf-EPSCs, $50 \mathrm{~ms}$ and $1 \mathrm{mV}$ for Mf-EPSPs. c, Summary graph of Mf-EPSC amplitudes (top left), charge transfer (top right, presented as mean \pm SEM, here and in all subsequent summary graphs), Mf-EPSP amplitudes (bottom left), and residual depolarization $65 \mathrm{~ms}$ after stimulation (bottom right) (WT: $n=9 ;$ GluK2 $^{-1-}: n=8 ; \mathrm{GluK3}^{-1-}: n^{-}$ 8; GluK5 ${ }^{-\prime-}: n=9 ;{ }^{* *} p<0.01$ and ${ }^{*} p<0.05$, compared with WT). Calibration: $10 \mathrm{ms,} 20$ pA.

and their output targets, the CA3 pyramidal cells by a conjunction of presynaptic and postsynaptic actions.

\section{Materials and Methods}

Mouse genotypes. Wild-type and mutant mice derived from heterozygote crossings of GluK2 $2^{-1-}$ (Mulle et al., 1998), GluK5 ${ }^{-/-}$(Contractor et al., 2003) and GluK1 ${ }^{-1-}$ (Mulle et al., 2000) mice were used in this study. These mice have been backcrossed on a C57BL6 background (for 11 generations). The GluK3 $3^{-1-}$ mice (Pinheiro et al., 2007) used have been backcrossed for 7 generations on a C57BL6 background.

Slice electrophysiology. Parasagittal brain slices (350 $\mu \mathrm{m}$ thick) were obtained from 2 to 3 week-old mice and were stored in an interface chamber for at least $1 \mathrm{~h}$ before transfer to a submersion recording chamber. The storage and perfusion solution contained (in $\mathrm{mm}$ ) $\mathrm{NaCl}$ (119), $\mathrm{KCl}(2.5), \mathrm{MgSO}_{4}$ (1.3), $\mathrm{CaCl}_{2}$ (2.5), $\mathrm{NaHCO}_{3}(26.2), \mathrm{NaH}_{2} \mathrm{PO}_{4}$ (1), and glucose (11), and was gassed with $95 \% \mathrm{O}_{2} / 5 \% \mathrm{CO}_{2}\left(23-25^{\circ} \mathrm{C}\right)$. Wholecell recordings were made from CA3 pyramidal cells under infrared differential interference contrast imaging at $33^{\circ} \mathrm{C}$. Picrotoxin $(100 \mu \mathrm{M})$ and

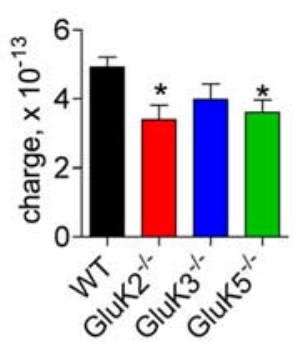

CGP55845 (5 $\mu \mathrm{M})$ were used to block GABA , and $\mathrm{GABA}_{\mathrm{B}}$ receptors respectively, unless mentioned otherwise. Experiments where the $I_{\text {sAHP }}$ activation was prevented were performed with an intracellular solution that contained (in $\mathrm{mM}$ ) Kgluconate (120 mM), KCl (20), HEPES (10), EGTA (10), NaATP (2), $\mathrm{MgCl}_{2}$ (2), $\mathrm{CaCl}_{2}$ (1). Experiments where $I_{\text {sAHP }}$ activation was allowed were performed with an intracellular solution that contained (in mM) Kgluconate (135 $\mathrm{mm}), \mathrm{NaCl}$ (8), HEPES (10), EGTA (0.2), $\operatorname{MgATP}(2), \mathrm{Na}_{3} \mathrm{GTP}(0.3)$. For both solutions, the $\mathrm{pH}$ was adjusted to $\mathrm{pH}$ 7.3. The access and input resistances were monitored throughout the experiments using a voltage or current step. Only cells with comparable input resistances were used (between 150 and $200 \mathrm{M} \Omega$ ). The access resistance was $<20 \mathrm{M} \Omega$ in whole-cell configuration and results were discarded if it changed by $>20 \%$. We observed no differences in the intrinsic excitability of CA3 pyramidal cells from all the genotypes (supplemental Fig. 1, available at www.jneurosci.org as supplemental material). A patch pipette filled with HEPES buffered external solution was placed in the hilus of the dentate gyrus to stimulate mossy fibers to evoke unitary Mf-EPSCs and EPSPs in CA3 pyramidal cells. A minimal stimulation protocol was used whereby the stimulating electrode was moved to a position where a sharp Mf-EPSC with minimal jitter was evoked. Stimulation intensity was adjusted just above the sharp threshold for activation of the synaptic response (see Fig. 1a). With such low stimulation intensity, no prominent polysynaptic activation was observed. Mf-EPSCs were recorded at $-70 \mathrm{mV}$ and corresponding MfEPSPs were obtained by switching to current clamp mode. Mossy fiber responses were characterized by the marked facilitation of EPSCs upon switching stimulus frequency from 0.1 to $1 \mathrm{~Hz}$, and by their sensitivity to the group II mGluR agonist L-CCG-1 (10 $\mu \mathrm{M})$ added routinely at the end of the experiment. For the induction of associative/commissural (A/C) long-term potentiation (LTP), a second stimulating electrode was placed in the CA3 stratum radiatum to activate the $\mathrm{A} / \mathrm{C}$ pathway. Recordings were made using an EPC 8.0 amplifier (HEKA Instruments). Signals were filtered at $0.5-1 \mathrm{kHz}$, digitized at $1-5 \mathrm{kHz}$, and stored on a personal computer for further analysis (IGOR PRO 5.0, Wavemetrics). Two physiological patterns of afferent input were used, with an average frequency of $1.4 \mathrm{~Hz}$ and $2.4 \mathrm{~Hz}$, each with a duration slightly $<30 \mathrm{~s}$. Each pattern was delivered in continuum (using IGOR PRO 5.0 waveform generator) and 10 consecutive traces for each pattern were used for the analysis. The patterns were kindly provided by Dr. Gyorgy Buzsaki (Rutgers University, Newark, NJ). All chemicals were obtained from Tocris Cookson or Sigma.

Statistical analysis. Values are presented as mean \pm SEM. Statistical comparisons were made using Student's unpaired $t$ test, and a post hoc Bonferroni test was used in the ANOVA analysis of the Mf-EPSP waveforms, and the analysis of spike number with respect to instantaneous stimulation frequency.

\section{Results}

The participation of KARs to excitatory synaptic currents at Mf synapses onto CA3 pyramidal cells has been characterized in detail (Lerma, 2006; Pinheiro and Mulle, 2006), but the physiological impact of KAR activation on the activity of CA3 neuronal 
circuitry remains undetermined. Here, we have examined how KARs control the short-term dynamics of synaptic integration and the efficacy of spike transmission between dentate granule cells and CA3 pyramidal cells. We have recorded mossy fiber excitatory synaptic potentials (MfEPSPs) from CA3 pyramidal cells in hippocampal slices from wild-type (WT) and selected KAR subunit-deficient mice in the absence of NMDA, AMPA, and KA receptor antagonists. Unless stated otherwise, recordings were performed with 10 mM EGTA in the patch pipette to prevent $I_{\text {sAHP }}$ activation, and in the presence of picrotoxin and CGP 55845 to block GABA receptors.

Throughout this study, a minimal stimulation protocol was used to evoke "unitary" Mf-EPSCs and Mf-EPSPs (Fig. 1a) (Jonas et al., 1993; Marchal and Mulle, 2004) (see Materials and Methods). Consistent with in vivo experiments (Henze et al., 2002), we found that single Mf-EPSPs do not drive spike discharge at low stimulation frequency whereas repetitive presynaptic activity increased spike transmission probability (see Figs. 1-4). To evaluate the function of presynaptic and postsynaptic KARs at the Mf-CA3 synapse in the absence of suitable specific antagonists, we have used WT and mice deficient for various KAR subunits. Because KARs are expressed early during postnatal development of CA3, we cannot rule out the possibility of compensatory changes, although there is yet no indication for this. Compensatory changes in GluK5 expression in GluK2 ${ }^{-1-}$ mice have indeed been reported to occur (Christensen et al., 2004; Ruiz et al., 2005). Nevertheless, we think that the comparisons between knock-out mice were meaningful as neither the intrinsic cellular parameters nor the basal amplitude of Mf-EPSPs were different between the four genotypes (Fig. 1; supplemental Fig. 1, available at www.jneurosci.org as supplemental material).

\section{Postsynaptic KARs shape Mf-EPSPs}

A single stimulation to mossy fibers is sufficient to activate synaptic KARs, although it only yields EPSCs of small amplitude with slow onset and decay kinetics (Castillo et al., 1997; Cossart et al., 2002). The relative amplitude of KAR-EPSCs, recorded under our experimental conditions in the presence of D-AP5 and GYKI $53655(50 \mu \mathrm{M})$, represented on average $5.2 \pm 0.2 \%(n=5)$ of Mf-EPSC amplitude, in close agreement with previous studies performed at room temperature (Castillo et al., 1997; Contractor et al., 2003; Marchal and Mulle, 2004; Ruiz et al., 2005). The physiological role of such a small amplitude ionotropic response mediated by KARs is questionable. However, due to its slow kinetics, the charge transfer through these receptors may have a significant role in shaping Mf-EPSPs.

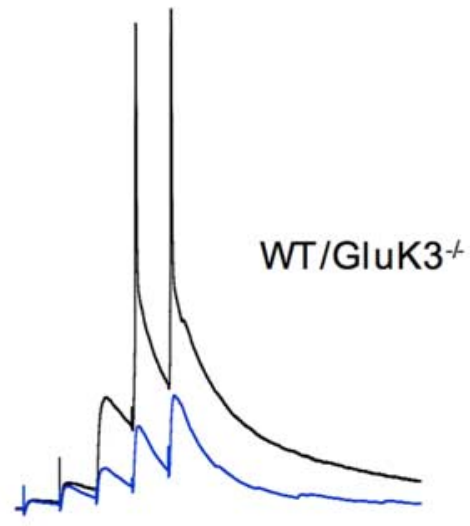

WT/GluK5+

GluK2-HGluK3*

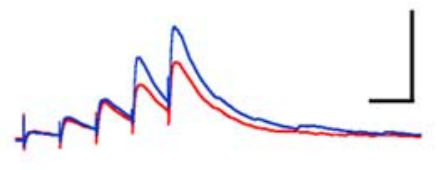

d
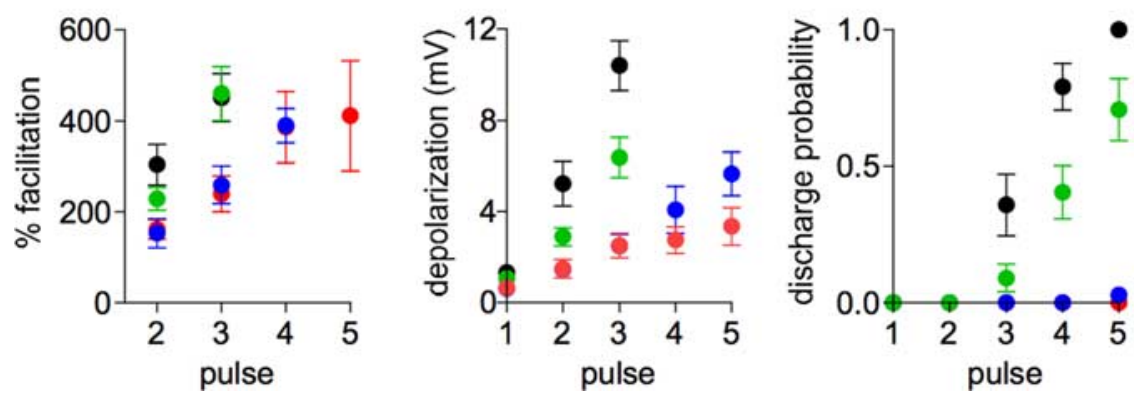

Figure 2. KARs facilitate spike transmission in response to short trains of stimulation. $\boldsymbol{a}$, Representative traces of Mf-EPSPs in response to a short $20 \mathrm{~Hz}$ stimulation train for WT, GluK2 ${ }^{-1-}$, GluK3 ${ }^{-1-}$, and GluK5 ${ }^{-1-}$ mice. $\boldsymbol{b}$, Summary graph of facilitation of Mf-EPSPs with respect to the amplitude of the first EPSP. c, Summary graph of residual depolarization of Mf-EPSPs. Residua depolarization was measured as the EPSP amplitude $50 \mathrm{~ms}$ after each stimulation. $\boldsymbol{d}$, Summary graph of discharge probability for each pulse of the $20 \mathrm{~Hz}$ train. The measurements for frequency facilitation and residual depolarization were obtained from cells that spiked only from the fourth pulse onwards for WT and GluK5 ${ }^{-1-}$, and values for the fourth and fifth pulse were omitted due to spiking. Consequently, WT and GluK5 ${ }^{-1-}$ values for the third pulse are probably slightly underestimated. Discharge probability was calculated using all the cells (WT: $n=6 ;$ GluK2 $\left.2^{-1-}: n=8 ; G_{l u K 3}{ }^{-1-}: n=6 ; G_{l u K 5}{ }^{-1-}: n=8\right)$. Calibration: 50 ms, $10 \mathrm{mV}$. Spikes were truncated in all traces.

We evaluated the impact of postsynaptic KAR activation on shaping Mf-EPSP waveform by analyzing GluK2 $2^{-1-}$ mice in which KAR-EPSCs are absent (Mulle et al., 1998). At low frequency $(0.1 \mathrm{~Hz})$, minimal stimulation of mossy fibers elicited Mf-EPSCs and Mf-EPSPs of comparable amplitudes in WT and GluK2 ${ }^{-1-}$ mice (Fig. $\left.1 b, c\right)$. However, the decay kinetics of MfEPSCs was faster in GluK2 ${ }^{-1-}$ mice, as illustrated by a decreased charge transfer (Fig. 1c). Consistently, ANOVA analysis of MfEPSP waveforms indicates that postsynaptic depolarization was significantly smaller in GluK2 ${ }^{-l-}$ than in WT mice between 28 and $121 \mathrm{~ms}$ after stimulation (Fig. $1 b, c$ ). We confirmed that MfEPSP waveform was shaped by postsynaptic KAR activation by analyzing these parameters in GluK3 ${ }^{-1-}$ mice, that only lack 
presynaptic KARs, and GluK5 ${ }^{-1-}$ mice, that display KAR-EPSCs with faster decay kinetics (Contractor et al., 2003) (Fig. 1). Accordingly, Mf-EPSCs and Mf-EPSPs decayed significantly faster in GluK5 ${ }^{-1-}$, but not in GluK3 ${ }^{-1-}$, compared with WT mice. Thus, the ionotropic response mediated by postsynaptic KARs contributes as a slow component that prolongs Mf-EPSPs, and can potentially play a role in the postsynaptic summation of the excitatory signals.

\section{KARs facilitate spike transmission}

In vivo, trains of stimulations are required to evoke spike transmission between granule cells and CA3 pyramidal cells (Henze et al., 2002). We thus examined the conditions required to trigger postsynaptic spikes with short trains of afferent stimulation in the acute slice preparation. In WT mice, synaptic facilitation induced during trains of 5 stimulations at $20 \mathrm{~Hz}$ systematically lead to spike discharge, the probability of which increased from the third to the fifth stimulation (Fig. 2). In contrast, in GluK2 ${ }^{-1-}$ mice that are devoid of both presynaptic and postsynaptic KARs, presynaptic facilitation and postsynaptic summation of Mf-EPSPs were greatly reduced, preventing spike threshold to be reached. (Fig. 2a,d). This was mainly due to the absence of KARdependent presynaptic facilitation, as in GluK3 ${ }^{-1-}$ mice a similar train of stimulation was also unable to trigger spike discharge with the exception of one cell (Fig. $2 a, d$ ). However, in both genotypes, prolongation of the train could lead to spike discharge (see Fig. 4a). The number of stimuli necessary to reach threshold was significantly smaller in GluK3 $3^{-1-}$ compared with GluK2 $2^{-1-}$ mice (see Fig. $4 d$ ), likely as a result of decreased postsynaptic summation in the latter (Fig. $2 a, c$ ). We also examined Mf-EPSPs in response to short trains of stimulations in $\mathrm{GluK5}^{-1-}$ mice, which display preserved levels of presynaptic facilitation but faster decaying KAR-EPSCs. Summation of Mf-EPSPs readily lead to spike discharge in GluK5 ${ }^{-1-}$ mice but with a significantly decreased probability when compared with WT mice $(p<0.05$, $n=8$, at third, fourth, and fifth pulse) (Fig. 2). We next evaluated whether the characteristic frequency facilitation properties of Mf synapses could lead to spiking. A single isolated presynaptic stimulation does not induce spike discharge in CA3 pyramidal cells (Fig. 3). Increasing tonic mossy fiber stimulation rate from 0.1 to 1 and $3 \mathrm{~Hz}$ produced robust facilitation of MfEPSCs (supplemental Fig. 2, available at www.jneurosci.org as supplemental material) and of Mf-EPSPs that eventually reached spike threshold (Fig. 3). Presynaptic KARs appear necessary to reach spike threshold under these conditions, although the slow postsynaptic component due to GluK2/ GluK5 heteromers may also contribute to spike transmission efficacy at $3 \mathrm{~Hz}$ (Fig. 3).

The delay between the onset of presynaptic stimulation and the first spike triggered in the postsynaptic cell depends on the number and frequency of presynaptic spikes. Setting this delay might be important for the precision of temporal coding in the hippocampal network (Henze et al., 2002). We analyzed how KARs influence this delay by applying trains of stimulation at frequencies ranging from $3 \mathrm{~Hz}$ to $100 \mathrm{~Hz}$ and measured the average delay between the onset of afferent activity and the onset of spike discharge in the postsynaptic target (Fig. 4). The delay to discharge, which decreases with the rate of stimulation, was markedly longer in GluK2 ${ }^{-1-}$ and GluK3 ${ }^{-1-}$ mice compared with WT mice at all frequencies up to $50 \mathrm{~Hz}$ (Fig. 4), and to a lesser degree in GluK5 ${ }^{-1-}$ mice up to $20 \mathrm{~Hz}$. No significant difference was observed for a train at $100 \mathrm{~Hz}$.
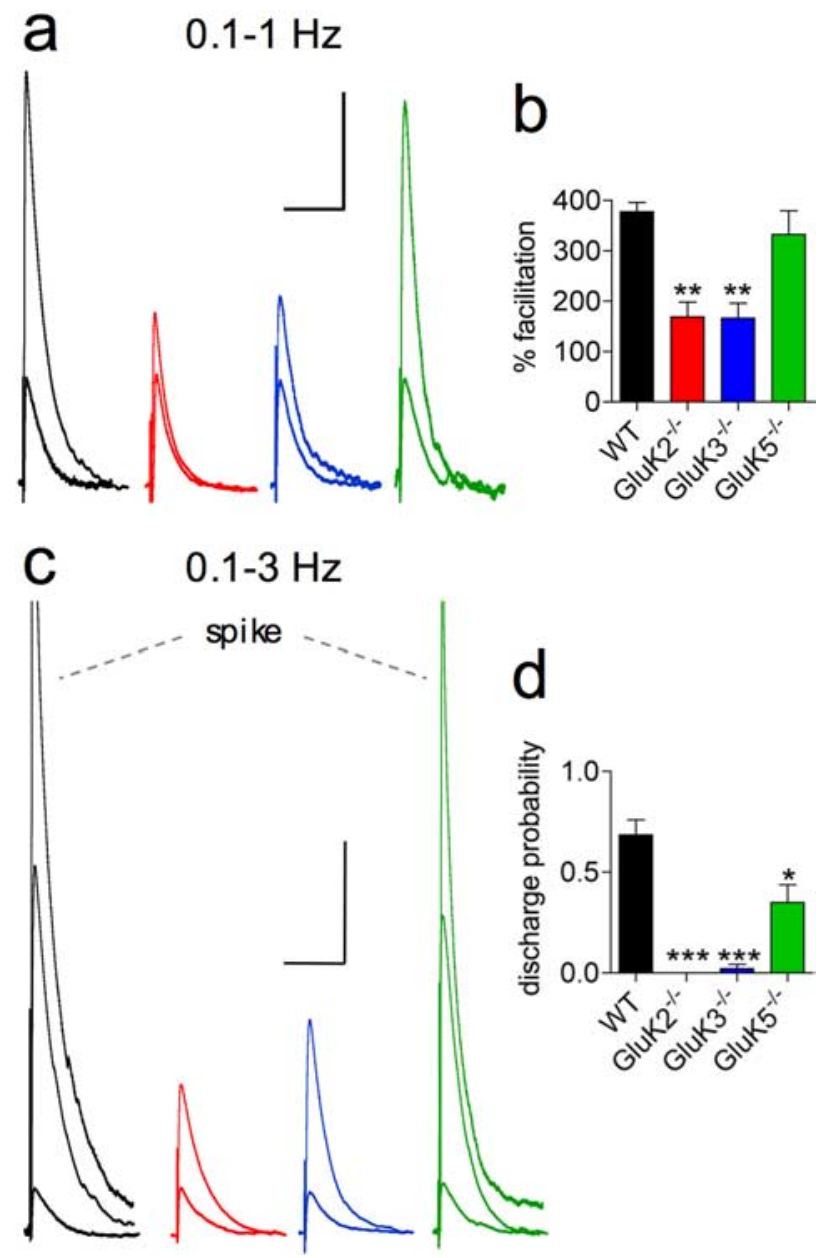

Figure 3. Presynaptic KARs are needed for spike discharge during frequency facilitation. $\boldsymbol{a}$, Traces illustrating frequency facilitation of Mf-EPSPs when shifting stimulation from 0.1 to $1 \mathrm{~Hz}$ for WT, GluK2 ${ }^{-1-}$, GluK3 ${ }^{-1-}$, and GluK5 ${ }^{-1-}$ mice. Superimposed traces are averaged from 15 and 40 recordings at 0.1 and $1 \mathrm{~Hz}$, respectively. $\boldsymbol{b}$, Summary graph of frequency facilitation from 0.1 to $1 \mathrm{~Hz}$ (WT: $n=8$; GluK2 ${ }^{-1-}: n=7$; GluK3 ${ }^{-1-}: n=6$; GluK5 ${ }^{-1-}: n=6$ ). c, Traces illustrating frequency facilitation of Mf-EPSPs from $0.1 \mathrm{~Hz}$ to $3 \mathrm{~Hz}$, for WT, GluK2 ${ }^{-1-}$, $\mathrm{GluK3}^{-1-}$ and GluK5 ${ }^{-1-}$ mice. Superimposed traces are averaged from 15 and 40 recordings at $0.1 \mathrm{~Hz}$ and $3 \mathrm{~Hz}$ respectively, for GluK2 $2^{-1-}$ and GluK3 ${ }^{-1-}$ mice, whereas the $3 \mathrm{~Hz}$ traces are averaged before spiking for WT and GluK5 ${ }^{-1-}$ mice. Both spikes are individual traces, and are truncated. $\boldsymbol{d}$, Summary graph of spike discharge probability in response to 60 mossy fiber stimulations at $3 \mathrm{~Hz}$ (WT: $0.65 \pm 0.07, n=7$; GluK2 ${ }^{-1-}: 0 \pm 0, n=6$; GluK3 $^{-1-}: 0.01 \pm$ $0.01, n=5$; GluK5 ${ }^{-1-}: 0.28 \pm 0.12, n=6$ ). The decreased spike discharge probability observed in GluK5 ${ }^{-1-}$ compared with WT mice is due both to the increased number of stimuli needed to reach threshold (14 \pm 2 vs $7 \pm 1$ ) (see also Fig. 4d) and to the decreased spike discharge probability after threshold was reached $(0.34 \pm 0.13$ vs $0.74 \pm 0.09)$. Calibrations: in $\boldsymbol{a}, 100 \mathrm{~ms}$ and $2 \mathrm{mV}$; in $\boldsymbol{c}, 100 \mathrm{~ms}$ and $5 \mathrm{mV}$. In $\boldsymbol{b}$ and $\boldsymbol{d},{ }^{*} p<0.05,{ }^{* *} p<0.01$, ${ }^{* * *} p<0.005$ compared with WT.

\section{GluK5 modulates mossy fiber spike transmission via $I_{\mathrm{sAHP}}$ regulation}

We have previously shown that synaptic activation of KARs in CA3 pyramidal cells inhibits the $\mathrm{Ca}^{2+}$-dependent $I_{\mathrm{sAHP}}$ through a GluK5-mediated metabotropic mechanism (Ruiz et al., 2005). To assess the role of this modulation on Mf-CA3 spike transmission, we applied an extended stimulation train of 40 pulses at $20 \mathrm{~Hz}$ in conditions that allowed the activation of $I_{\mathrm{sAHP}}$ and its inhibition by synaptic KARs (i.e., with $0.2 \mathrm{~mm}$ EGTA in the patch pipette instead of 10 mM) (Ruiz et al., 2005). Effectively, an $I_{\text {sAHP }}$ of $52 \pm 11 \mathrm{pA}(n=11)$ was obtained in the presence of $0.2 \mathrm{~mm}$ EGTA, but it was systematically $<5 \mathrm{pA}(n=7)$ with $10 \mathrm{~mm}$ EGTA in the patch pipette. Inter- 
estingly, preventing $I_{\text {sAHP }}$ activation with 10 mM EGTA increased the probability of spike transmission within the train to a value close to 1 , consistent with the implication of a $\mathrm{Ca}^{2+}$-dependent ionic conductance in the regulation of excitability. The probability of spike discharge was largely reduced in GluK5 ${ }^{-1-}$ mice (Fig. 5) $(p<0.005$ compared with WT), indicating that synaptic activation of GluK5-containing KARs increase spike transmission during sustained presynaptic stimulation by controlling the excitability of postsynaptic pyramidal cells.

\section{KARs facilitate induction of associative/} commissural long-term potentiation

As presynaptic and postsynaptic KARs at the Mf synapse facilitate spike transmission in CA3 pyramidal cells, they may favor spike timing-dependent plasticity. The overlap between mossy fiber and A/C fiber activity has been recently shown to induce such a form of plasticity at the A/C synapse (Kobayashi and Poo, 2004), where KARs do not contribute directly to the EPSCs. We therefore investigated the implication of KARs at the Mf synapse in its induction. LTP was induced at the A/C synapse in both WT and GluK2 $2^{-/-}$mice with simultaneous paired stimulation trains to the mossy fibers ( 5 pulses, $50 \mathrm{~Hz}$ ) and A/C fibers $(8$ pulses, $50 \mathrm{~Hz})$, at $1 \mathrm{~s}$ intervals, repeated 10 times (Kobayashi and Poo, 2004). The resulting LTP (30 min after induction) at $\mathrm{A} / \mathrm{C}$ synapses was greatly reduced in GluK2 ${ }^{-1-}$ mice, compared with WT mice (Fig. 6a,b,f). LTP was however not abrogated in GluK2 $2^{-1-}$ mice, where a progressive run up of A/C EPSPs was observed. In WT and GluK2 ${ }^{-1-}$ mice, where only trains of A/C tetanus were given in the absence of MF stimulation, we observed a slow and modest run up of the A/C EPSPs, $\sim 20$ min after the tetanus (Fig. $6 a, b$ ). This run up was absent in baseline conditions, where no tetanus trains were applied (data not shown). Hence it seems that the slow run up is a property of the A/C synapse, induced after the application of tetanus stimulation. In addition, a subthreshold depolarization induced by a train of Mf-EPSPs might also contribute to the slowly developing LTP of A/C synapses observed in GluK2 ${ }^{-1-}$ mice (Fig. $6 b, e$ ). LTP of A/C synapses was reduced to similar levels in GluK3 ${ }^{-/-}$mice (Fig. $6 c, e$ ), indicating that presynaptic KARs indirectly impact the induction of LTP. In both these genotypes, impairment of LTP correlates with a reduction in the number of APs evoked during the induction protocol compared with WT mice (Fig. $6 f$ ). LTP of A/C afferents was only marginally impaired in GluK5 $5^{-/-}$mice (Fig. $6 d, e)$, consistent with the lack of significant decrease in spike number during the induction protocol (Fig. $6 f$ ). These experiments indicate that KARs at mossy fiber synapses contribute to an associative form of LTP at A/C afferents mainly by its ability to amplify spike transmission during the induction protocol.
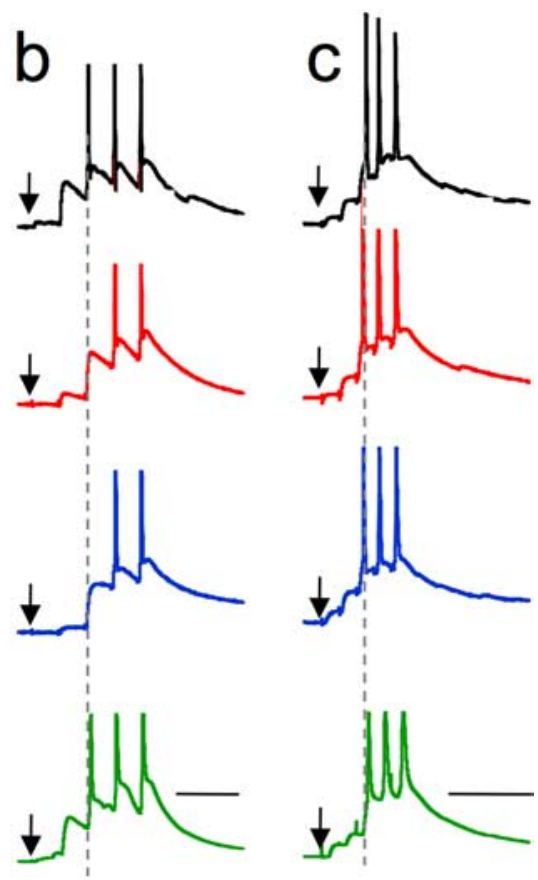

Figure 4. KARs shorten the delay to reach spike discharge. $\boldsymbol{a}-\boldsymbol{c}$, Representative Mf-EPSP traces in response to $20 \mathrm{~Hz}(\boldsymbol{a}), 50 \mathrm{~Hz}$ (b), and $100 \mathrm{~Hz}$ stimulation trains (c) for WT, GluK2 ${ }^{-1-}$, GluK3 ${ }^{-1-}$, and GluK5 ${ }^{-1-}$ mice. The arrows point to the first stimutraces. $\boldsymbol{d}$, Summary graphs of the delay to trigger the first spike at $3 \mathrm{~Hz}$ (WT: $n=7$; GluK5 ${ }^{-1-}: n=6$ ), $20 \mathrm{~Hz}$ (WT: $n=6$; $n=4)$, and $100 \mathrm{~Hz}$ (WT: $n=5$; GluK2 ${ }^{-1-}: n=4$; GluK3 ${ }^{-1-}: n=4 ;$ GluK5 $\left.^{-1-}: n=4\right)\left({ }^{*} p<0.05,{ }^{* *} p<0.01,{ }^{* * *} p<0.001\right.$ compared with WT). Calibrations: in $\boldsymbol{a}, 200 \mathrm{~ms}$ and $20 \mathrm{mV}$; in $\boldsymbol{b}, \boldsymbol{c}, 50 \mathrm{~ms}$ and $20 \mathrm{mV}$. Spikes were truncated in all traces.

\section{KARs determine CA3 pyramidal cell output in response to physiological patterns of stimulation}

The previous experiments were performed by applying calibrated patterns of stimulation of mossy fibers whereas granule cells of the dentate gyrus have been reported to display a wide range of discharge frequencies (Jung and McNaughton, 1993). These patterns of neuronal activity recorded in vivo can be used as an input to stimulate mossy fibers. We used two such patterns recorded from place cells in the dentate gyrus of freely moving mice (see Materials and Methods). Each pattern of stimulation (30 s duration) was repeated in continuum in the presence of intact glutamatergic and GABAergic transmission, and $0.2 \mathrm{~mm}$ EGTA in the recording pipette, allowing conditions to be as close to physiological as possible. For both patterns of stimulation used, GluK2 ${ }^{-1-}$ mice displayed a markedly altered output pattern with a lower spike discharge probability, compared with WT mice (Fig. 7; supplemental Fig. 4, available at www.jneurosci.org as supplemental material). Analyzing spike discharge probability as 


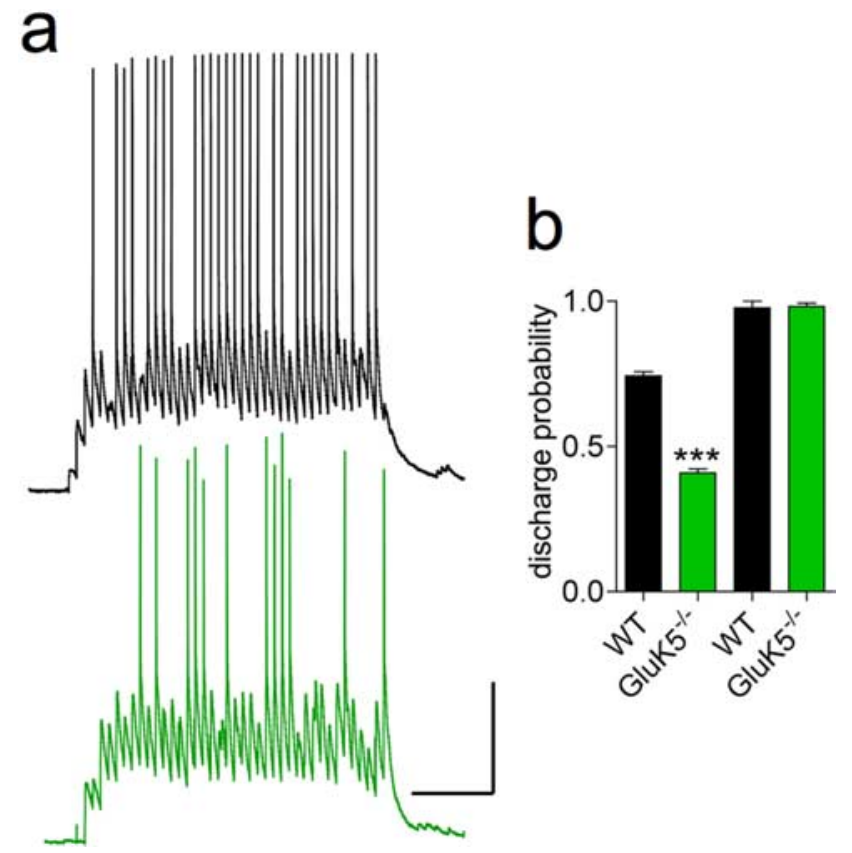

Figure 5. KARs enhances spike discharge probability via GluK5. $\boldsymbol{a}$, Representative recordings in response to a prolonged $20 \mathrm{~Hz}$ stimulation train, with $0.2 \mathrm{~mm}$ EGTA in the patch pipette to preserve $I_{\text {sAHP, }}$ for WT (top) and GluK5 ${ }^{-I-}$ mice (bottom). Note the difference in the number of stimulations needed before spike discharge in GluK5 ${ }^{-1-}$ compared with WT mice. $\boldsymbol{b}$, Summary graph of the spike discharge probability, with and without intact $I_{\text {sAHP, }}$ for WT ( $n=11$ and $n=$ 6 , respectively) and GluK5 ${ }^{-1-}$ mice ( $n=9$ and $n=8$, respectively). The discharge probability is calculated after the first spike in the train. Representative recordings in the absence of $I_{\text {sAHP }}$ are presented in Figure $4 a .{ }^{* * *} p<0.005$ compared with WT). Calibration: $500 \mathrm{~ms}, 20 \mathrm{mV}$. Spikes were truncated in all traces.

a function of instantaneous frequency of presynaptic stimulation revealed that it increased progressively in WT mice whereas only frequencies $>10 \mathrm{~Hz}$ evoked spikes in GluK2 ${ }^{-1-}$ mice. The probability of spike discharge for each afferent stimulus was markedly smaller at all instantaneous frequencies in GluK2 ${ }^{-1-}$ mice and this difference was statistically significant from WT mice for the two patterns for instantaneous stimulation frequencies ranging from 1 to $50 \mathrm{~Hz}$ (Fig. 7d).

The discharge probability for the entire pattern duration was also reduced in GluK3 ${ }^{-1-}$ mice compared with WT mice (Fig. $7 c$ ), the relationship between discharge probabilities and instantaneous frequency of presynaptic stimulation being intermediate between that of GluK2 ${ }^{-1-}$ and WT mice (Fig. $7 d$ ). The role of the GluK1 subunit appears to be minimal, if any, on spike transmission at MF-CA3 synapses, as we observed no differences in spike outcome between WT and GluK1 ${ }^{-1-}$ mice in response to the physiological patterns of stimulation (supplemental Fig. 5, available at www.jneurosci.org as supplemental material). GluK5 ${ }^{-1-}$ mice also displayed a lower probability of spike discharge compared with WT mice $(p<0.01)$ (Fig. $7 c, d)$. This decrease can, at least in part, be attributed to the metabotropic modulation of neuronal excitability by synaptic KARs containing the GluK5 subunit. These results highlight the essential role of KARs in ensuring the efficacy of spike transmission during physiological patterns of afferent activity.

\section{Discussion}

The present experiments provide a direct demonstration of a role for KARs in the efficacy of spike transmission at Mf-CA3 synapses. These receptors act as "conditional" amplifiers of spike transmission at synapses between granule cells and CA3 pyramidal cells in response to physiological patterns of activity. Although KARs were predicted to participate in the dynamics of synaptic integration and information transfer at Mf-CA3 synapses (Nicoll and Schmitz, 2005; Pinheiro and Mulle, 2006), this had not yet received direct experimental support. We show that KARs amplify synaptic integration and spike transmission within a wide range of afferent stimulation frequencies (from 1 to $50 \mathrm{~Hz}$ ) thus facilitating the induction of associative LTP at A/C synapses. KARs also reduce the delay between the onset of granule cell activity and target neuron activation and hence, decrease the threshold for information transfer at Mf-CA3 synapses. By controlling the short-term dynamics of Mf synaptic transmission, KARs allow Mf synapses to function as conditional detonators.

\section{Presynaptic and postsynaptic receptors concur to amplify spike transmission}

The differential subunit composition of presynaptic and postsynaptic KARs at Mf-CA3 synapses (GluK2/GluK3 presynaptic, GluK2/GluK5 postsynaptic), together with the availability of mice with distinct impairment in KAR function, offer the invaluable possibility to understand the mechanisms by which KARs amplify synaptic signals and spike transmission at this synapse. The analysis of GluK3 ${ }^{-1-}$ mice, which only exhibit a presynaptic phenotype (Pinheiro et al., 2007), reveals that presynaptic KARs play a prominent role in this process at all frequencies ranging from $1 \mathrm{~Hz}$ to $50 \mathrm{~Hz}$. A recent study has challenged the idea that presynaptic kainate receptors are involved in the facilitation of synaptic transmission at the mossy fiber-CA3 synapse, arguing that the contribution to facilitation instead arose from polysynaptic activation of synapses driven by postsynaptic kainate receptors (Kwon and Castillo, 2008). A major argument against this possibility is the fact that presynaptic facilitation is markedly decreased in GluK3 $3^{-1-}$ mice, whereas this subunit does not participate in postsynaptic EPSCs in CA3 pyramidal cells (Pinheiro et al., 2007) (this study). The reason for a lack of difference between wild-type and GluK3 ${ }^{-1-}$ mice reported in their study can be attributed to the fact that the concentrations of extracellular divalent cations used $\left(4 \mathrm{mMCa}^{2+} / 4 \mathrm{mM} \mathrm{Mg}^{2+}\right.$ ) mask the activity of presynaptic KARs [Pinheiro et al. (2007); see also the study by Lauri et al. (2003)]. Under conditions of minimal stimulation intensity, EPSC rise time values (supplemental Fig. $1 f$, available at www.jneurosci.org as supplemental material) were similar to that reported by Kwon and Castillo (2008) in the presence of low TTX concentrations. In addition, we have previously reported that decreasing the amplitude of AMPA-EPSCs by $>70 \%$ with low concentrations of NBQX (250 nM) affects neither frequency facilitation nor paired-pulse facilitation of Mf-EPSCs (Perrais et al., 2009). These data strongly argue against the contribution of polysynaptic activity to presynaptic facilitation, at least in our experimental conditions.

Postsynaptic KARs, by prolonging Mf-EPSPs, also contribute (although to a lesser extent) to synaptic integration and spike transmission as indicated by the comparison of GluK3 ${ }^{-1-}$ and GluK2 ${ }^{-1-}$ mice. This small but slow postsynaptic component is amplified by KAR-dependent presynaptic facilitation. In support of the participation of KAR-EPSCs to synaptic integration [this study, see also the studies by Frerking and Ohliger-Frerking (2002), Goldin et al. (2007) in CA1 interneurons] a moderate reduction of KAR-EPSC amplitude by synaptic activation of NMDA receptors leads to substantial changes in the waveform of Mf-EPSPs (Rebola et al., 2007). Finally this study provides a direct demonstration that the postsynaptic metabotropic action of 
KARs has a substantial impact on spike transmission in physiological conditions of stimulation, as indicated by the analysis of GluK5 $5^{-/-}$mice. Because this effect concerns the inhibition of a slowly activating $\mathrm{K}^{+}$conductance, its impact is best observed following sustained activity. Long stimulation trains and physiological stimulation patterns led to markedly decreased spike discharge probability in GluK5 ${ }^{-1-}$ mice compared with WT mice. Blocking $I_{\text {sAHP }}$ prevented this GluK5 mediated effect and revealed that, under physiological conditions, the portion of $I_{\mathrm{sAHP}}$ unaffected by GluK5 is a potential target for further modulation.

\section{Functional relevance for integrated functions}

The efficacy of Mf synaptic transmission can vary to a large extent depending on the pattern of afferent activity, in striking difference with A/C synapses onto CA3 pyramidal cells which only display modest facilitation (Salin et al., 1996). The exact prediction of how a specific pattern of activity in presynaptic granule cells is translated into an output pattern of spike activity is difficult to assess, given the variety of presynaptic and postsynaptic mechanisms that interplay. Nonetheless, repetitive stimulation with the same complex train mimicking physiological activity yielded highly reproducible responses (Fig. 7), indicating that the code for spike transmission is precise.

In this study we have not taken into account the potential role of feedforward inhibition. In cultured hippocampal slices, a single action potential in a granule cell was shown to evoke a biphasic response in a CA3 pyramidal cell, consisting of an EPSC followed by an IPSC (Mori et al., 2004). This secondary inhibitory response likely reflects feedforward inhibition due to disynaptic inhibition from interneurons connected by mossy fibers in CA3 stratum lucidum (Acsády et al., 1998; Maccaferri et al., 1998). Interestingly, when GABAergic synaptic transmission was kept intact in our acute slice experiments, minimal stimulation only occasionally led to biphasic (excitatory-inhibitory) postsynaptic responses. Furthermore, we did not find any significant differences in spike transmission obtained with or without intact GABAergic transmission (supplemental Fig. 3, available at www.jneurosci.org as supplemental material). This suggests that, contrary to slice cultures, monosynaptic excitation and feedforward inhibition from a single granule cell do not usually target the same CA3 pyramidal neuron in acute slices. Hence, the possibility that in acute slices lateral inhibition predominates over feedforward inhibition needs to be further
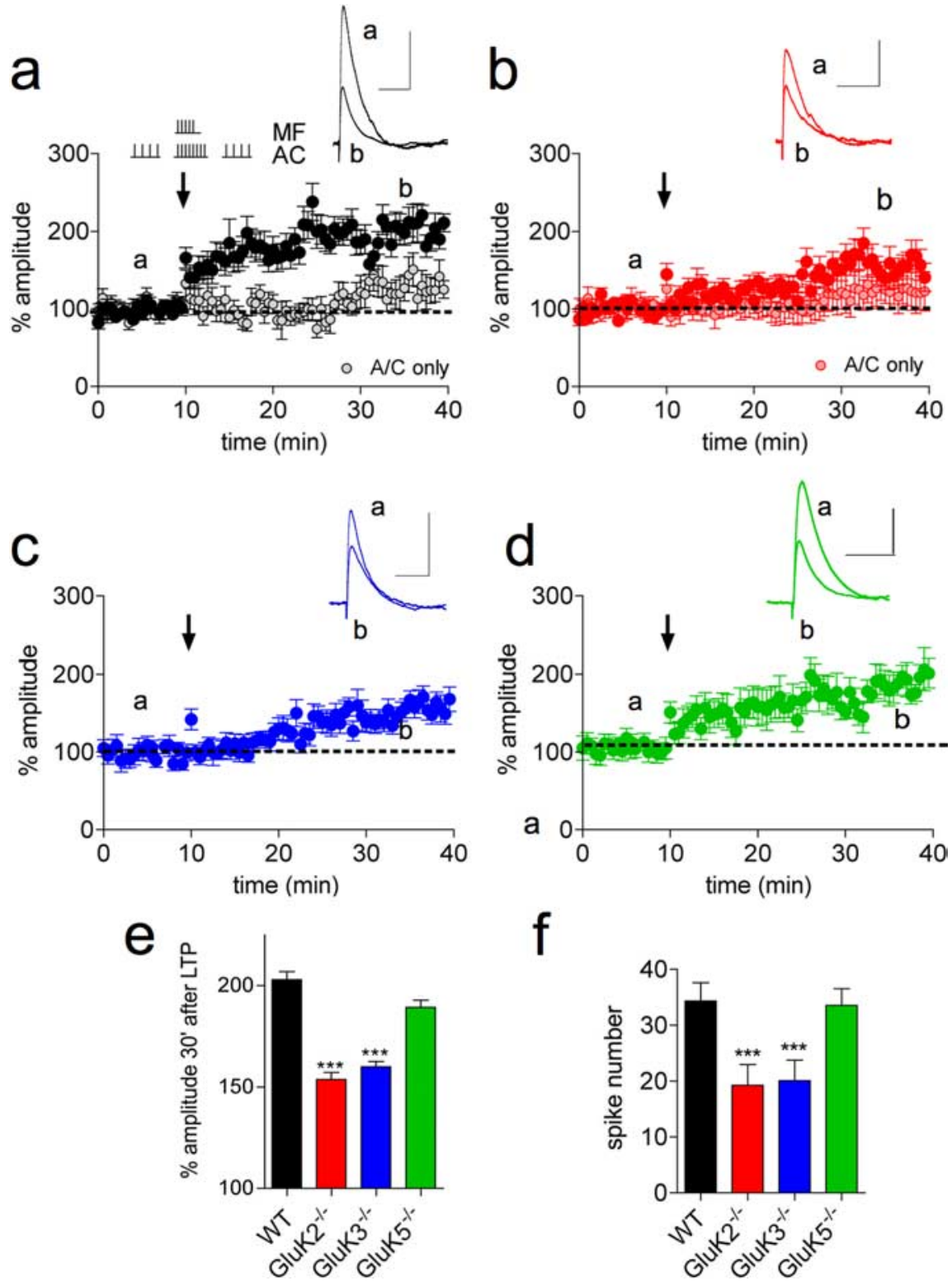

Figure 6. KARs facilitate the induction of A/C LTP. $a, A / C L T P$ was induced by a coincident pairing protocol of stimulation trains to the mossy fibers ( 5 pulses, $50 \mathrm{~Hz}$ ) and $\mathrm{A} / \mathrm{C}$ fibers ( 8 pulses, $50 \mathrm{~Hz}$ ), as illustrated. This pairing protocol was repeated 10 times at $1 \mathrm{~Hz}$ at the time indicated by the arrow. A/C fibers were otherwise stimulated at $0.1 \mathrm{~Hz}$ and EPSP amplitudes were averaged every minute and normalized to the mean amplitude of the 10 min preceding the LTP pairing protocol. Right, Traces represent A/CEPSPS before (a) and after LTP induction (b). A/C only graphs, The gray (a, WT) and the pink (b, GluK2 ${ }^{-1-}$ ) circles correspond to A/CEPSP amplitudes in conditions where the LTP-inducing train was only delivered to $\mathrm{A} / \mathrm{C}$ afferents in the absence of a conditioning train to mossy fibers. $\boldsymbol{b}-\boldsymbol{d}$, As in $\boldsymbol{a}$, but in GluK2 ${ }^{-1-}$, GluK3 ${ }^{-1-}$, and GluK5 ${ }^{-1-}$ mice. $\boldsymbol{e}$, LTP was significantly diminished 30 min after LTP induction in GluK2 $2^{-1-}$ and GluK3 ${ }^{-1-}$ mice compared with WT mice (WT: $204 \pm 6 \%, n=16$; GluK2 ${ }^{-1-}: 155 \pm 6, n=15$, ${ }^{* * *} p<0.001$; GluK3 $\left.{ }^{-1-}: 159 \pm 5 \%, n=11,{ }^{* * *} p<0.001\right)$ and to a small extent in GluK5 ${ }^{-1-}$ mice $(190 \pm 6 \%, n=13, p=$ 0.114). $f$, A higher number of spikes were evoked during the induction protocol in WT and GluK5 ${ }^{-1-}$ mice, compared with GluK2 $^{-1-}$ and GluK3 ${ }^{-1-}$ mice (WT: $34 \pm 3, n=16$; GluK2 ${ }^{-1-}: 19 \pm 4, n=15^{* * *} p<0.01$; GluK3 ${ }^{-1-}: 20 \pm 4, n=11$, ${ }^{* * *} p<0.01$; GluK5 $\left.{ }^{-1-}: 34 \pm 3, n=13, p=0.858\right)$. Calibration: $100 \mathrm{~ms}$ and $2 \mathrm{mV}$ for the EPSPs.

investigated. Additionally, because of the distinct presynaptic properties of synapses made onto interneurons and pyramidal cells, the inhibitory response depresses while the excitatory response facilitates with increasing presynaptic afferent activity (Acsády et al., 1998; Mori et al., 2004). Feedforward inhibition between mossy fibers and CA3 pyramidal cells is certainly important in designing the temporal code with which spike transmission is made possible. The expression of KARs in CA3 interneurons suggests the possible involvement of 


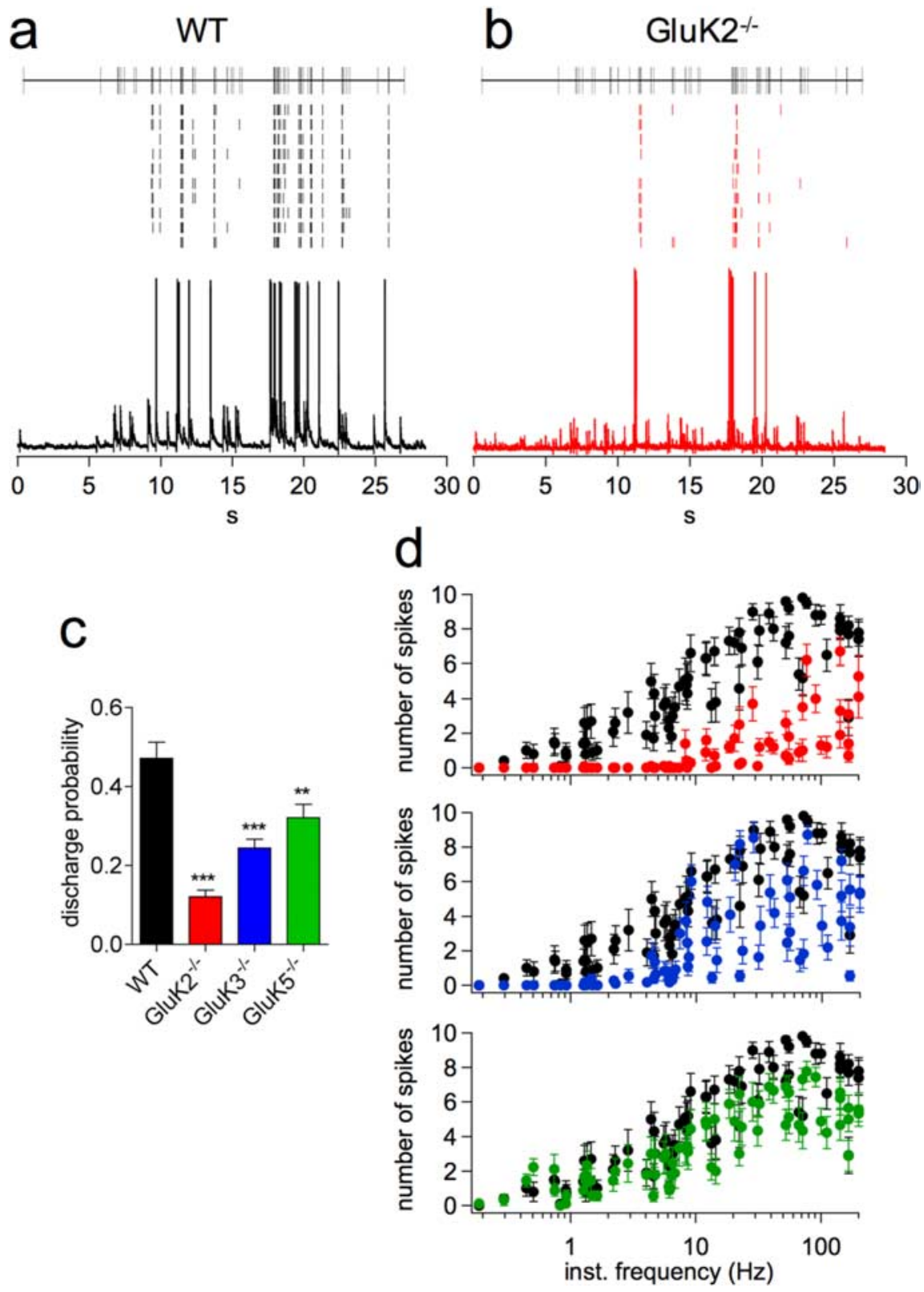

Figure 7. KARs control CA3 pyramidal cell output in response to a physiological granule cell firing pattern of stimulation. $\boldsymbol{a}, \boldsymbol{b}$, Raster plots of spike discharge from 10 consecutive recordings (top) in response to the physiological pattern illustrated at the top of the panel, with representative recordings (bottom). This pattern of stimulation was extracted from an in vivo recording of a granule cell from the dentate gyrus of a freely moving mouse. Data are from a single CA3 pyramidal cell of a WT $(\boldsymbol{a})$ and a GluK2 $2^{-I-}$ mouse (b). c, Probability of spike discharge during physiological pattern of stimulation for WT ( $\left.n=16\right)$, GluK2 ${ }^{-1-}$ $(n=19)$, GluK3 ${ }^{-I-}(n=19)$, and GluK5 ${ }^{-I-}(n=18)$ mice. Data were pooled from the $30-$--long patterns of stimulation presented here and in supplemental Figure 4 , available at www.jneurosci.org as supplemental material. $\boldsymbol{d}$, Plots of the number of spikes during 10 consecutive recordings as a function of the instantaneous frequency of each stimulation for WT $(n=10)$ and GluK2 ${ }^{-1-}(n=10)$, GluK3 $^{-1-}(n=11)$, and GluK5 ${ }^{-1-}(n=9)$ mice for the pattern at $2.4 \mathrm{~Hz}\left({ }^{* *} p<0.01,{ }^{* * *} p<0.001\right.$ compared to WT).

these receptors in regulating feedforward inhibition at Mf synapses (Mulle et al., 2000; Paternain et al., 2000), but this has yet to be addressed. It is worth mentioning that our data using a physiological pattern of presynaptic activity in slices from WT mice share large similarities with what has been reported in vivo, in conditions where a single presynaptic granule cell drives the firing of a postsynaptic CA3 pyramidal cell (Henze et al., 2002) and where feedforward inhibition should be operant. Although local network activity likely modulate the ef- ficacy of information transfer, the basic features of spike transmission at MFCA3 synapses are largely determined by the intrinsic properties of this synapse and, thus by KARs.

Models of hippocampal function predict that the mossy fiber synapse is critical for enabling episodic memories to be formed and stored in the CA3 network (Rolls and Kesner, 2006). There has been recent experimental support for different functions of CA3 and CA1 subfields in the processing of spatial information (Guzowski et al., 2004), but experimental evidence for a specific role of mossy fiber synapses in these processes are scarce. For instance, it has been proposed that the characteristic properties of mossy fiber synapses may be important for pattern completion and pattern separation (Bischofberger et al., 2006), but this has not been directly tested, for instance by targeted inactivation of synaptic transmission (Nakashiba et al., 2008).

KARs at Mf-CA3 synapses play a critical role in providing a "Hebbian" postsynaptic depolarization that conditions the long-lasting strengthening of $\mathrm{A} / \mathrm{C}$ inputs, thus the activity of the auto-associative CA3 network. Due to its extensive network of recurrent collaterals, the CA3 area is proposed to be capable of retrieving entire spatial patterns from partial or degraded inputs (Rolls and Kesner, 2006). Hence, completion of degraded inputs may thus implicate KARs at Mf-CA3 synapses. Previous behavioral analysis of GluK2 ${ }^{-1-}$ mice have shown that the mutant mice expressed reduced contextual and auditory fear memory (Ko et al., 2005), but appeared normal when exposed to a gross behavioral test battery, including the Morris water maze (Mulle et al., 1998). As the GluK2 subunit is widely expressed in the brain, the analysis of mice with a constitutive deletion of GluK2 makes it difficult to establish a clear link between behavioral deficits and electrophysiological impairments in CA3 pyramidal cells. Nevertheless, our findings call for a reevaluation of spatial memory in GluK2 $2^{-1-}$ mice, such as in behavioral paradigms that require the mice to perform pattern completion tasks (Nakazawa et al., 2004). These behavioral paradigms would be especially fruitful if applied to mice with a conditional deletion of KARs in the hippocampal CA3 subfield.

\section{References}

Acsády L, Kamondi A, Sík A, Freund T, Buzsáki G (1998) GABAergic cells are the major postsynaptic targets of mossy fibers in the rat hippocampus. J Neurosci 18:3386-3403.

Bischofberger J, Engel D, Frotscher M, Jonas P (2006) Timing and efficacy 
of transmitter release at mossy fiber synapses in the hippocampal network. Pflugers Arch 453:361-372.

Castillo PE, Malenka RC, Nicoll RA (1997) Kainate receptors mediate a slow postsynaptic current in hippocampal CA3 neurons. Nature 388:182-186.

Christensen JK, Paternain AV, Selak S, Ahring PK, Lerma J (2004) A mosaic of functional kainate receptors in hippocampal interneurons. J Neurosci 24:8986-8993.

Contractor A, Swanson G, Heinemann SF (2001) Kainate receptors are involved in short- and long-term plasticity at mossy fiber synapses in the hippocampus. Neuron 29:209-216.

Contractor A, Sailer AW, Darstein M, Maron C, Xu J, Swanson GT, Heinemann SF (2003) Loss of kainate receptor-mediated heterosynaptic facilitation of mossy-fiber synapses in KA2 ${ }^{-1-}$ mice. J Neurosci 23:422-429.

Cossart R, Epsztein J, Tyzio R, Becq H, Hirsch J, Ben-Ari Y, Crépel V (2002) Quantal release of glutamate generates pure kainate and mixed AMPA/ kainate EPSCs in hippocampal neurons. Neuron 35:147-159.

Frerking M, Ohliger-Frerking P (2002) AMPA receptors and kainate receptors encode different features of afferent activity. J Neurosci 22:7434-7443.

Goldin M, Epsztein J, Jorquera I, Represa A, Ben-Ari Y, Crépel V, Cossart R (2007) Synaptic kainate receptors tune oriens-lacunosum moleculare interneurons to operate at theta frequency. J Neurosci 27:9560-9572.

Guzowski JF, Knierim JJ, Moser EI (2004) Ensemble dynamics of hippocampal regions CA3 and CA1. Neuron 44:581-584.

Henze DA, Urban NN, Barrionuevo G (2000) The multifarious hippocampal mossy fiber pathway: a review. Neuroscience 98:407-427.

Henze DA, Wittner L, Buzsáki G (2002) Single granule cells reliably discharge targets in the hippocampal CA3 network in vivo. Nat Neurosci 5:790-795.

Jonas P, Major G, Sakmann B (1993) Quantal components of unitary EPSCs at the mossy fiber synapse on CA3 pyramidal cells of the rat hippocampus. J Physiology 472:615-663.

Jung MW, McNaughton BL (1993) Spatial selectivity of unit activity in the hippocampal granular layer. Hippocampus 3:165-182.

Ko S, Zhao MG, Toyoda H, Qiu CS, Zhuo M (2005) Altered behavioral responses to noxious stimuli and fear in glutamate receptor 5 (GluR5)- or GluR6-deficient mice. J Neurosci 25:977-984.

Kobayashi K, Poo MM (2004) Spike train timing-dependent associative modification of hippocampal CA3 recurrent synapses by mossy fibers. Neuron 41:445-454.

Kwon HB, Castillo PE (2008) Role of glutamate autoreceptors at hippocampal mossy fiber synapses. Neuron 60:1082-1094.

Lauri SE, Bortolotto ZA, Nistico R, Bleakman D, Ornstein PL, Lodge D, Isaac JT, Collingridge GL (2003) A role for Ca2+ stores in kainate receptordependent synaptic facilitation and LTP at mossy fiber synapses in the hippocampus. Neuron 39:327-341.

Lerma J (2006) Kainate receptor physiology. Curr Opin Pharmacol 6:89-97.
Maccaferri G, Tóth K, McBain CJ (1998) Target-specific expression of presynaptic mossy fiber plasticity. Science 279:1368-1370.

Marchal C, Mulle C (2004) Postnatal maturation of mossy fibre excitatory transmission in mouse CA3 pyramidal cells: a potential role for kainate receptors. J Physiol 561:27-37.

Melyan Z, Wheal HV, Lancaster B (2002) Metabotropic-mediated kainate receptor regulation of IsAHP and excitability in pyramidal cells. Neuron 34:107-114.

Mori M, Abegg MH, Gähwiler BH, Gerber U (2004) A frequencydependent switch from inhibition to excitation in a hippocampal unitary circuit. Nature 431:453-456.

Mulle C, Sailer A, Pérez-Otaño I, Dickinson-Anson H, Castillo PE, Bureau I, Maron C, Gage FH, Mann JR, Bettler B, Heinemann SF (1998) Altered synaptic physiology and reduced susceptibility to kainate-induced seizures in GluR6-deficient mice. Nature 392:601-605.

Mulle C, Sailer A, Swanson GT, Brana C, O'Gorman S, Bettler B, Heinemann SF (2000) Subunit composition of kainate receptors in hippocampal interneurons. Neuron 28:475-484.

Nakashiba T, Young JZ, McHugh TJ, Buhl DL, Tonegawa S (2008) Transgenic inhibition of synaptic transmission reveals role of CA3 output in hippocampal learning. Science 319:1260-1264.

Nakazawa K, McHugh TJ, Wilson MA, Tonegawa S (2004) NMDA receptors, place cells and hippocampal spatial memory. Nat Rev Neurosci 5:361-372.

Nicoll RA, Schmitz D (2005) Synaptic plasticity at hippocampal mossy fibre synapses. Nat Rev Neurosci 6:863-876.

Paternain AV, Herrera MT, Nieto MA, Lerma J (2000) GluR5 and GluR6 kainate receptor subunits coexist in hippocampal neurons and coassemble to form functional receptors. J Neurosci 20:196-205.

Perrais D, Pinheiro PS, Jane DE, Mulle C (2009) Antagonism of recombinant and native GluK3-containing kainate receptors. Neuropharmacology 56:131-140.

Pinheiro P, Mulle C (2006) Kainate receptors. Cell Tissue Res 326:457-482.

Pinheiro PS, Perrais D, Coussen F, Barhanin J, Bettler B, Mann JR, Malva JO, Heinemann SF, Mulle C (2007) GluR7 is an essential subunit of presynaptic kainate autoreceptors at hippocampal mossy fiber synapses. Proc Natl Acad Sci U S A 104:12181-12186.

Rebola N, Sachidhanandam S, Cunha RA, Mulle C (2007) Short-term plasticity of kainate receptor mediated EPSCs induced by NMDA receptors at hippocampal mossy fiber synapses. J Neurosci 27:3987-3993.

Rolls ET, Kesner RP (2006) A computational theory of hippocampal function, and empirical tests of the theory. Prog Neurobiol 79:1-48.

Ruiz A, Sachidhanandam S, Utvik JK, Coussen F, Mulle C (2005) Distinct subunits in heteromeric kainate receptors mediate ionotropic and metabotropic function at hippocampal mossy fiber synapses. J Neurosci 25:11710-11718

Salin PA, Scanziani M, Malenka RC, Nicoll RA (1996) Distinct short-term plasticity at two excitatory synapses in the hippocampus. Proc Natl Acad Sci U S A 93:13304-13309. 